\title{
Shielding design of the Mayo Clinic Scottsdale cyclotron vault
}

\author{
Kenneth A. Van Riper ${ }^{1, *}$, Robert L. Metzger ${ }^{2}$, and Kevin Nelson ${ }^{3}$ \\ ${ }^{1}$ White Rock Science, P. O. Box 4729, Los Alamos, NM 87547, USA \\ ${ }^{2}$ Radiation Safety Engineering, 3245 N. Washington, Chandler, AZ 85225, USA \\ ${ }^{3}$ Scottsdale Mayo Clinic, 13400 E. Shea Blvd., Scottsdale,AZ 85259, USA
}

\begin{abstract}
Mayo Clinic Scottsdale (Scottsdale, Arizona) is building a cyclotron vault containing a cyclotron with adjacent targets and a beam line leading to an external target. The targets are irradiated by high energy (15 to $16.5 \mathrm{MeV}$ ) protons for the production of radioisotopes. We performed Monte Carlo radiation transport simulations to calculate the radiation dose outside of the vault during irradiation of the cyclotron and external targets. We present the Monte Carlo model including the geometry, sources, and variance reduction methods. Mesh tallies surrounding the vault show the external dose rate is within acceptable limits.
\end{abstract}

\section{Introduction}

A cyclotron facility is under construction at the Scottsdale campus of the Mayo Clinic. The General Electric (GE) cyclotron's primary use is for production of ${ }^{18} \mathrm{~F}$ for PET imaging. The PET isotopes are produced in a target adjacent to the accelerator. The proton beam can be directed to an external target in an adjoining room for production of other clinical and research isotopes including ${ }^{11} \mathrm{C},{ }^{13} \mathrm{~N},{ }^{15} \mathrm{O},{ }^{63} \mathrm{Zn}$, and ${ }^{68} \mathrm{Ga}$. We refer to the former as the cyclotron target and the latter as the external target.

\section{Monte Carlo Model}

\subsection{Geometry}

The geometry model consists of the entrance maze, the cyclotron room containing the cyclotron target and collimator material, and the external target room with the external target. Details of the cyclotron are not included; we assume the shielding supplied by the vendor will reduce the leakage of any internal radiation from the accelerator to values low in comparison to the sources we consider: a fractional loss of the proton beam passing through a collimator, the interaction of the beam with the target, and the photons emitted by ${ }^{18} \mathrm{~F}$.

Figure 1 shows a floor plan of the model overlaid on an architect's drawing of the vault. Thick pink lines outline the Monte Carlo regions except for the interior and exterior air spaces. The concrete walls and ceiling are 6 feet thick. The floor is 3 feet thick. An air space approximately $31 / 2$ feet ( 1.1 meter) thick surrounds the vault above the floor level. Below the floor line, the exterior consists of concrete.

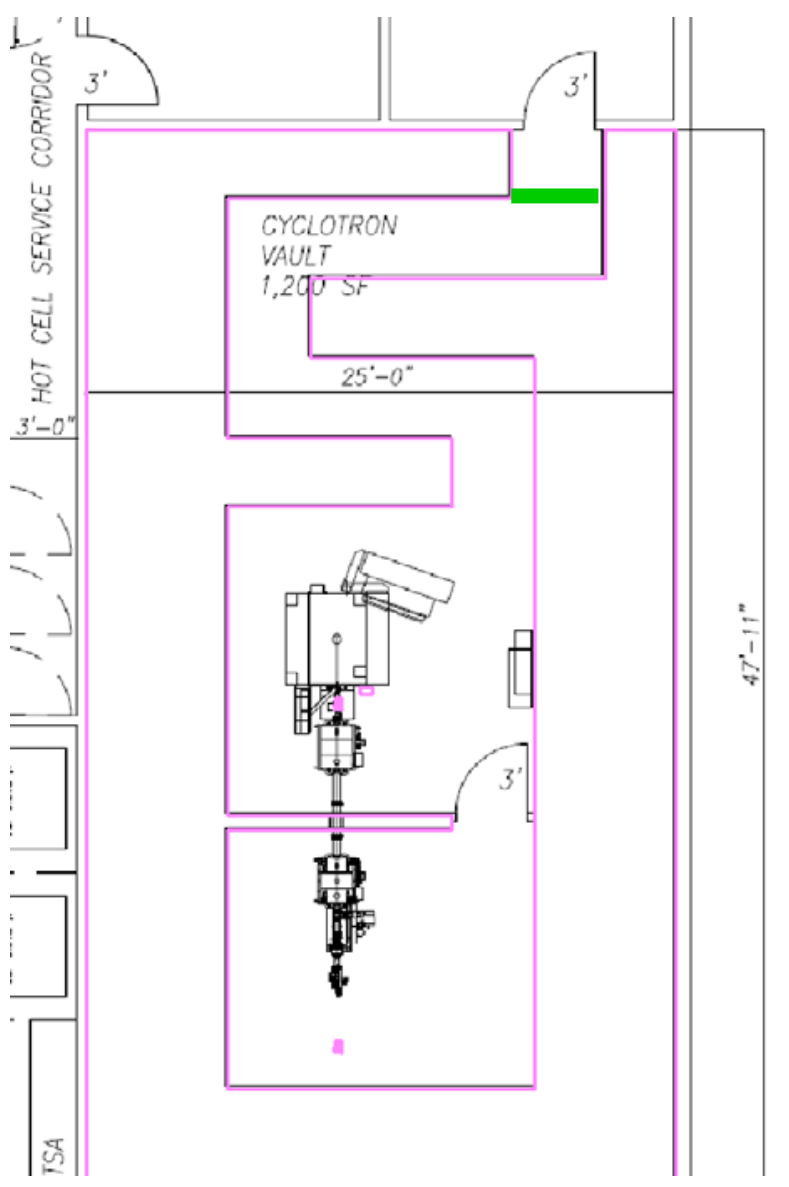

Figure 1. Monte Carlo model overlaid on the architectural drawing.

Also shown in pink in Figure 1 are the outlines of the targets and the collimator as modeled. The external target is located further from the end of the beam line shown in the drawing. The exact location will not affect

\footnotetext{
* Corresponding author: kvr@rt66.com
} 
the radiation produced and its penetration through the walls.

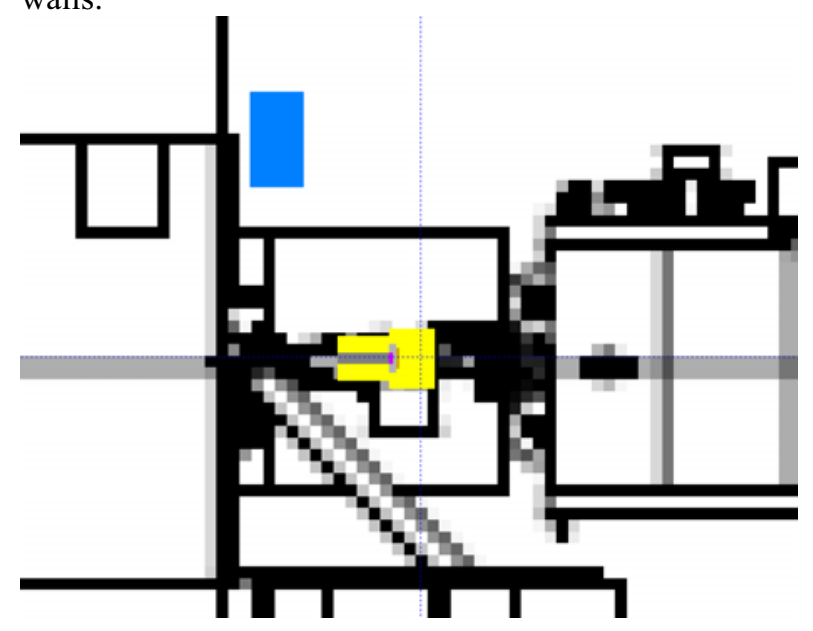

Figure 2. Detail of Figure 1 showing the cyclotron target and collimator block.

Figure 2 shows an enlarged section of Figure 1 around the cyclotron target. The blue block offset from the beam line is a graphite cube used to model the beam fraction (assumed to be $10 \%$ ) that is lost when passing though the collimator. It is offset from the beam line to prevent any particles that penetrate the block from reaching the target. We refer to this block as the collimator even though it is not a detailed model of that component. The cyclotron and beam line components were not included in the model.

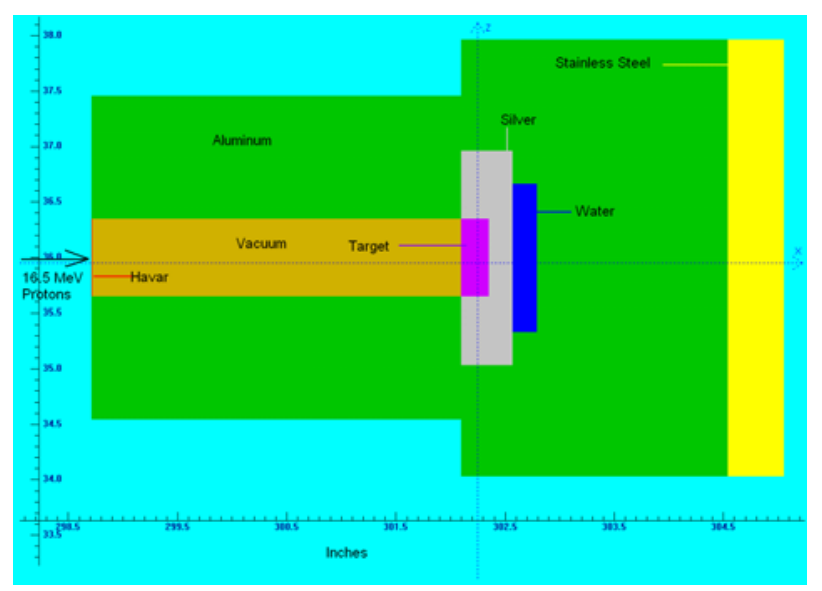

Figure 3. Target Model

Figure 3 shows the target model. It was adapted from the GE PETTRACE document[1]. The same model is used in both target locations. All components are concentric cylinders centered 36 inches above the floor. The dimensions are in inches. A beam of $16.5 \mathrm{MeV}$ protons is incident on the $75 \mu \mathrm{M}$ thick Havar foil covering a vacuum (brown in Fig. 3) downstream from the target. The target (magenta) is ${ }^{18} \mathrm{O}$ enriched water. It is surrounded upstream by a silver backing plate (grey) with cooling water further upstream. These components are encased in aluminum (green) and stainless steel (yellow).

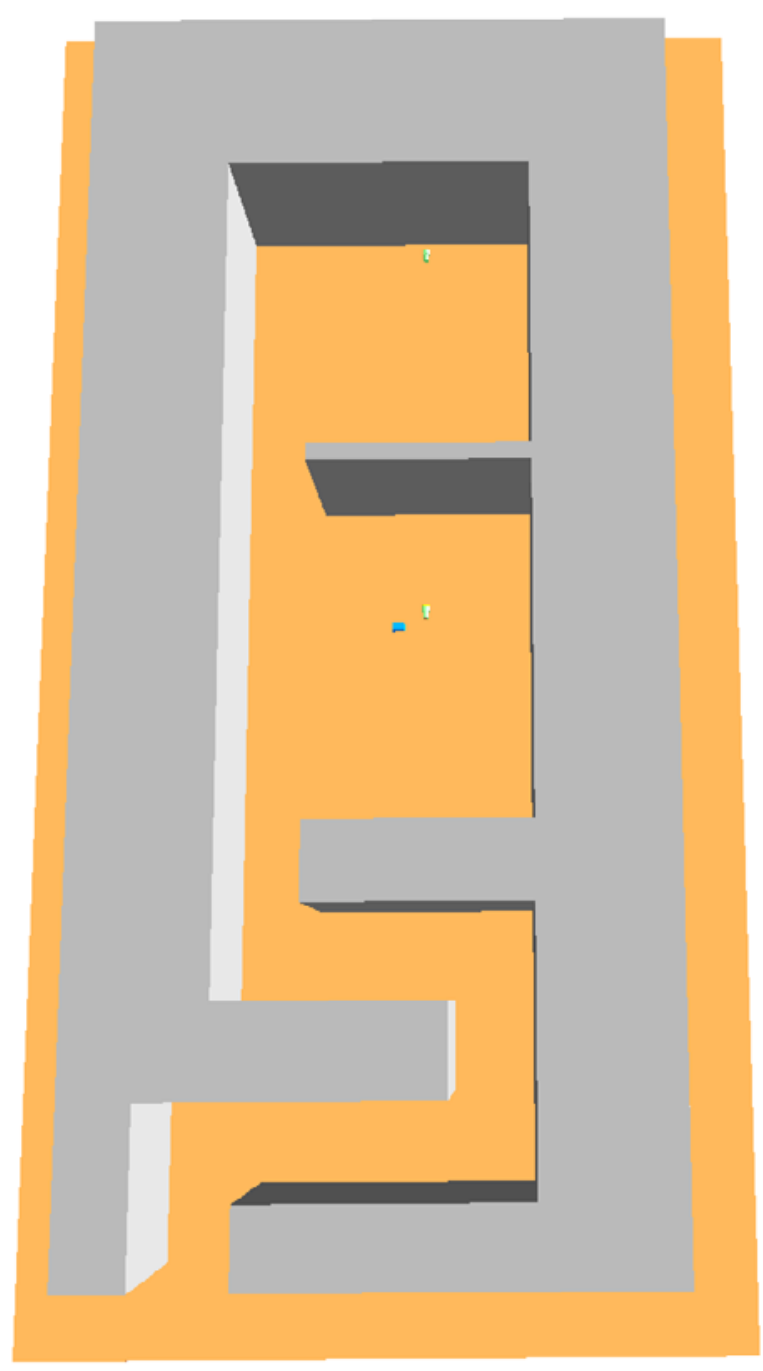

Figure 4. 3D Cutaway view of the cyclotron vault.

Figure 4 is a 3 dimensional (3D) view of the cyclotron vault with the ceiling cut away. The air is invisible, the concrete walls are gray, and the concrete floor is orange. The targets are yellow and the collimator block is blue.

\subsection{Source terms}

The cyclotron emits a beam of $16.5 \mathrm{MeV}$ protons. The maximum current used in the cyclotron room is $130 \mu \mathrm{A}$ (micro Amperes) after a 10\% loss upon passing through the collimator. The $130 \mu \mathrm{A}$ is split into $65 \mu \mathrm{A}$ on two targets. For the Monte Carlo model, we use $130 \mu \mathrm{A}$ on a single target. We also assume a fully irradiated target emitting $2 \times 3500 \mathrm{mCi}$ of $0.511 \mathrm{MeV}$ photons. Table 1 summarizes the cyclotron room target source components. The total rate of emission is $8.927 \times 10^{14}$ particles / second. 


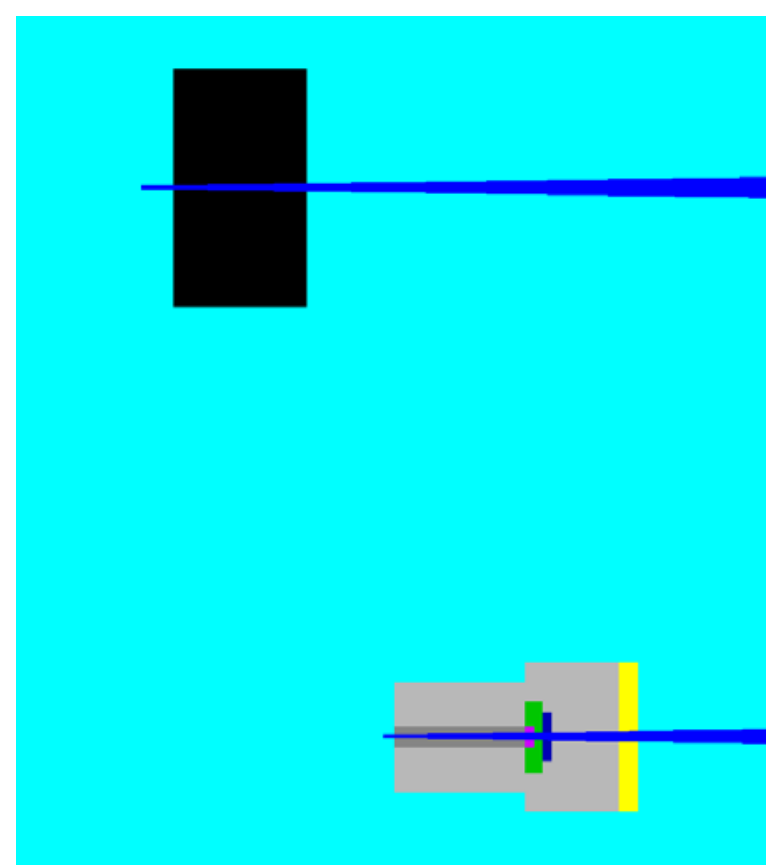

Figure 5. Proton beams (dark blue) striking collimator (top) and cyclotron target (bottom).

Two proton beams are directed towards the target and collimator with a small divergence angle. The beam origins are just downstream of the target and collimator. Figure 5 shows the two beams for the cyclotron room model. The figure is from a test run with no interactions; with interactions, the beams are stopped in the target and collimator.

Table 1. Source components for cyclotron target model.

\begin{tabular}{|l|l|l|l|}
\hline Component & Particle & $\begin{array}{l}\text { Energy } \\
(\mathrm{MeV})\end{array}$ & Particles / Second \\
\hline $130 \mu \mathrm{A}$ on Target & Proton & 16.5 & $8.1133 \times 10^{14}$ \\
\hline $\begin{array}{l}13 \mu \mathrm{A} \text { on Collimator } \\
(10 \% \text { loss })\end{array}$ & Proton & 16.5 & $8.1133 \times 10^{13}$ \\
\hline $7000 \mathrm{mCi}$ Photons & Photon & 0.511 & $2.59 \times 10^{11}$ \\
\hline
\end{tabular}

The photons are emitted isotropically. The photon source is distributed uniformly throughout the target water $\left(\mathrm{H}_{2}{ }^{18} \mathrm{O}\right)$ volume.

Table 2. Source components for the external target model

\begin{tabular}{|l|l|l|l|}
\hline Component & $\begin{array}{l}\text { Particl } \\
\mathrm{e}\end{array}$ & $\begin{array}{l}\text { Energy } \\
(\mathrm{MeV})\end{array}$ & Particles / Second \\
\hline $80 \mu \mathrm{A}$ on Target & Proton & 16.5 & $4.9928 \times 10^{14}$ \\
\hline $\begin{array}{l}8 \mu \mathrm{A} \text { on Collimator } \\
(10 \% \text { loss })\end{array}$ & Proton & 16.5 & $4.993 \times 10^{13}$ \\
\hline $3500 \mathrm{mCi}$ Photons & Photon & 0.511 & $1.255 \times 10^{11}$ \\
\hline
\end{tabular}

The target room model assumes a $80 \mu \mathrm{A}$ proton beam impinging on the target in the external target room, a 8 $\mu \mathrm{A}$ beam loss on the collimator in the cyclotron room, and single $3500 \mathrm{mCi}$ load of irradiated target material.
Table 2 summarizes the source components. The total rate of emission is $5.493 \times 10^{14}$ particles / second.

\subsection{Mesh tallies}

The results are shown in the form of mesh tallies where the dose equivalent rate and its statistical error (relative error in Monte Carlo parlance) are calculated in each cell of a mesh overlaid on the geometry. Each mesh cell in the plane of the mesh is approximately $5 \mathrm{~cm}$ square and is $50 \mathrm{~cm}$ thick. Each mesh tally extends 1 meter outside of the vault. There are separate mesh tallies for the neutron, photon, and proton dose equivalent rates. These three were summed in post processing to give the total dose equivalent rate. Figures 6 and 7 show the mesh tally locations.

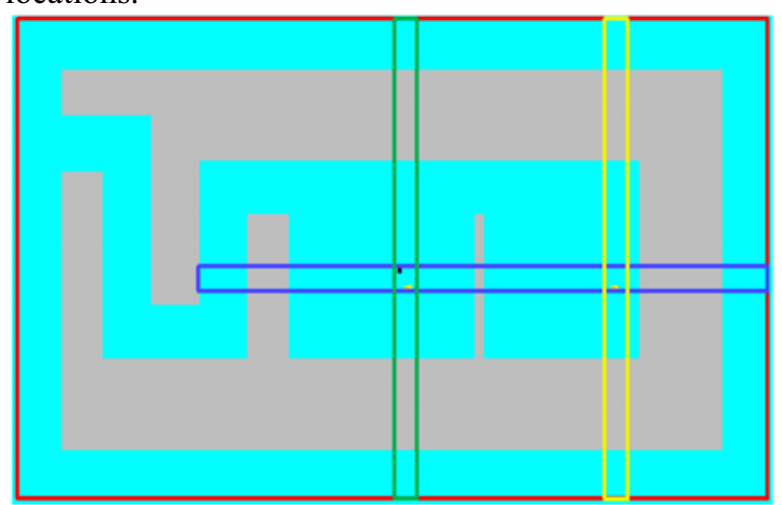

Figure 6. Mesh tally locations in the XY floor plan view.

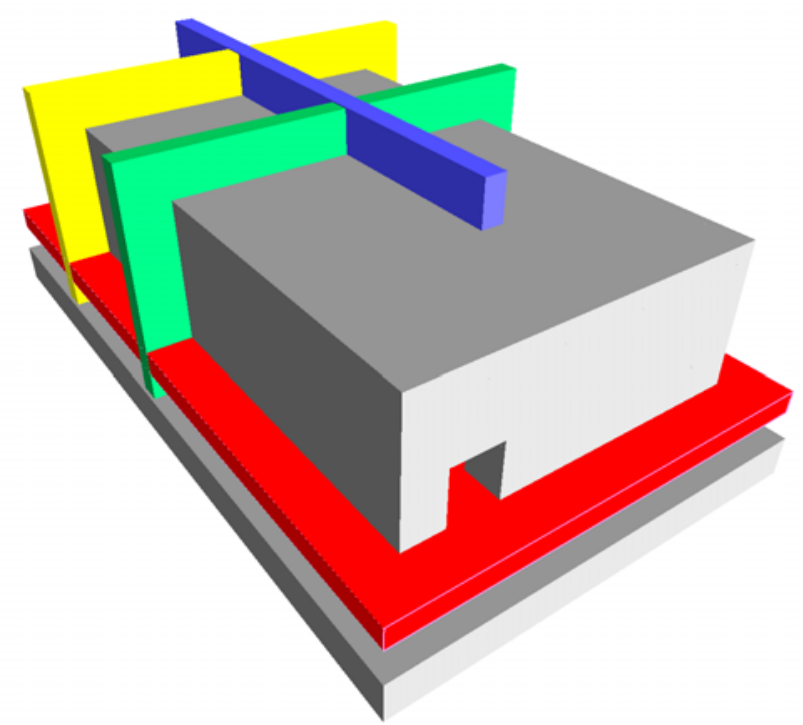

Figure 7. Mesh tally locations in the $3 \mathrm{D}$ view.

The red mesh tally covers the entire model, centered on the height of the targets and collimator. The vertical dark blue tally also passes through the targets and collimator. The red and blue tallies are used by both models (cyclotron targer and external target). The dark green tally is used in the cyclotron room target model and passes through the cyclotron target and collimator. The dark green tally is not used in the external target model. That model uses the yellow mesh tally that passes through the external target. 


\subsection{Monte Carlo items}

The transport of protons, neutrons, and photons was calculated with the Monte Carlo code MCNPX version 2.7.0 [2].

Dose response functions are used to convert the calculated flux, in particles $/ \mathrm{cm}^{2} / \mathrm{second}$, to a dose equivalent rate in $\mathrm{Sv} /$ hour. The dose equivalent is the absorbed dose at a point in tissue weighted by a distribution of quality factors related to the LET distribution of radiation at that point. For neutrons, the response function is taken from NCRP-38 1971, ANSI/ANS 6.1.1-1977. The quality factors in the NCRP-38 dose response function match those listed in 10 CFR Part 20. For photons, the values in ICRP-21 1971 are used. For protons, the values given in a NASA report were used.

Variance reduction, or biasing, is required to calculate penetration of the thick walls and ceiling and to direct the radiation through the entrance maze. We used the technique of importance splitting. When a particle traverses from a region of lower to high importance, it is split into two equivalent particles so that there is a greater chance of one of those particles avoiding absorption. The model uses 26 importance layers through the walls and ceiling and 31 layers between the source and entrance.

A calculation was run for each of the two source configurations. Each followed at least $5 \times 10^{7}$ source particles. The cyclotron target model had some large (> $0.1)$ relative errors in the external target room and far wall. An additional cyclotron target model with biasing adjusted towards the external target room was run to correct those deficiencies. Combined mesh tallies show the mesh cells with the lowest relative error from the two cyclotron target runs.

\section{Vault shielding results}

The mesh tally results are presented as color wash figures overlaid with an outline of the geometry. Contours are shown at $1 \times 10^{-2}, 1 \times 10^{-3}, 1 \times 10^{-4}, 1 \times 10^{-5}$, $1 \times 10^{-6}, 1 \times 10^{-7}$, and $1 \times 10^{-8} \mathrm{~Sv} /$ hour. Values greater than $100 \mathrm{~Sv} / \mathrm{hr}$ are colored red. Values less than $10^{-10}$ $\mathrm{Sv} / \mathrm{hr}$ are dark blue. Different color schemes are used for the dose rate and for the relative error.

\subsection{Cyclotron target model}

Figures $8,9,10$, and 11 show the neutron, photon, proton, and total dose equivalent rates, respectively, in the horizontal mesh tally for the cyclotron target model. External to the vault, the neutrons and photons give nearly equal contributions to the total. The only significant proton contribution is near the target and collimator from protons in the beam and protons scattered off components along the beam line. Figure 10 shows the beams are completely stopped in the target and collimator. Additional protons are produced by interactions in the concrete. These secondary protons contribute a very small fraction to the total dose rate.

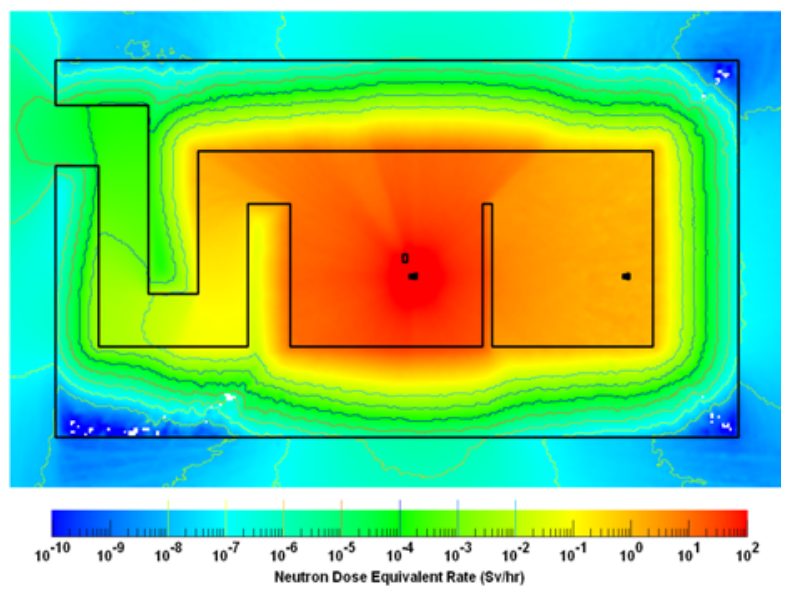

Figure 8. Neutron dose equivalent rate in the horizontal mesh tally of the cyclotron target model.

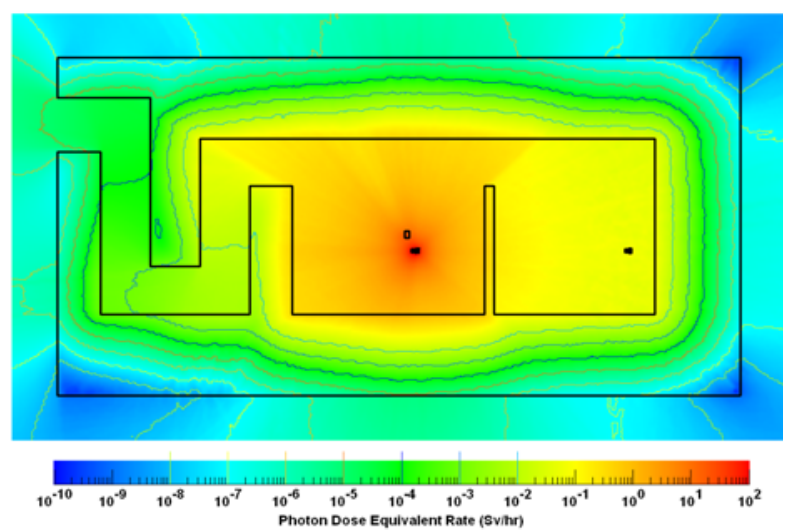

Figure 9. Photon dose equivalent rate in the horizontal mesh tally of the cyclotron target model.

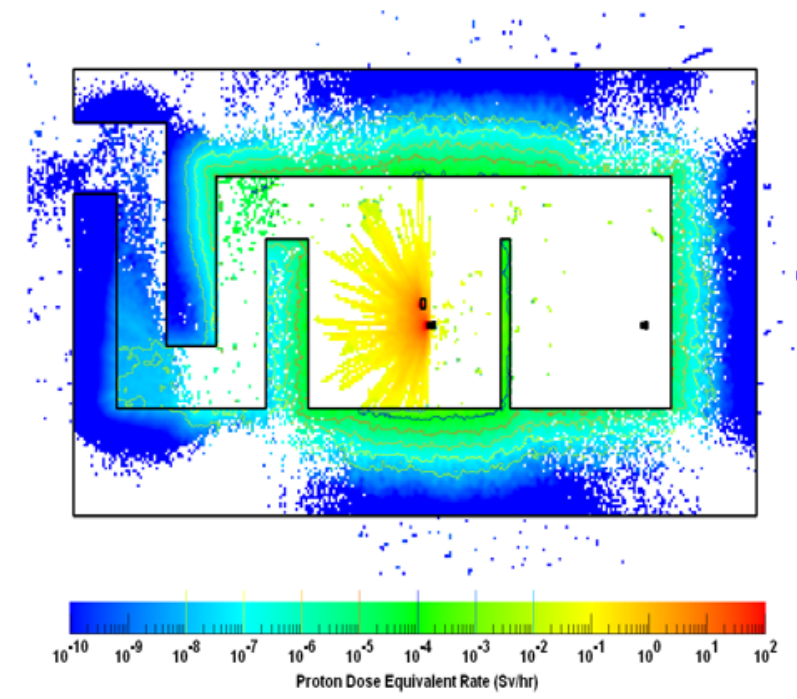

Figure 10. Proton dose equivalent rate in the horizontal mesh tally of the cyclotron target model.

Figure 12 shows the relative error for the total dose rate. Except for some regions within the walls, the relative error is less than 0.1 in areas of interest, an indication that the results are valid.

Figure 13 plots the dose equivalent rates extracted from the mesh tallies of Figures 8 through 10 along a line from top to bottom passing through the target and collimator. The proton contribution peaks at the target and collimator. 


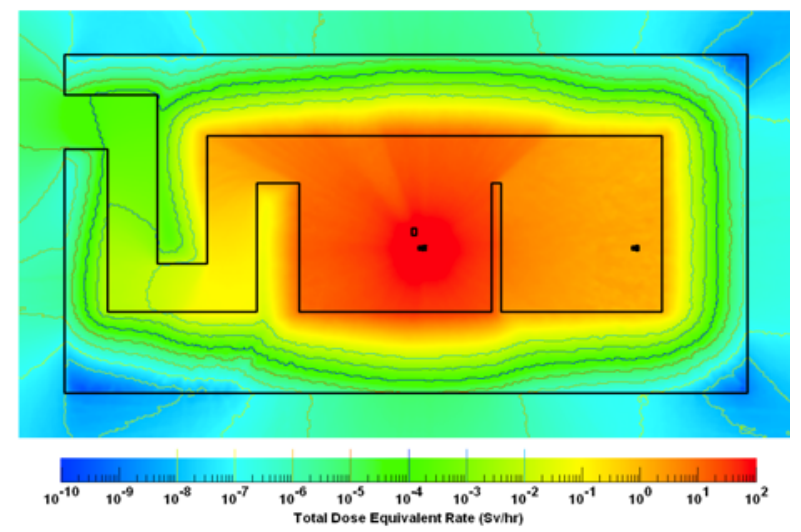

Figure 11. Total dose equivalent rate in the horizontal mesh tally of the cyclotron target model.
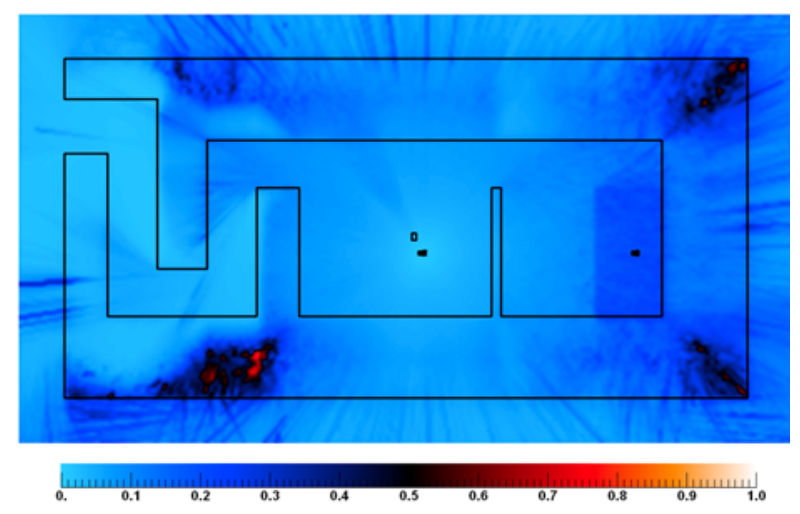

Total Dose Relative Error

Figure 12. Total dose equivalent rate relative error in the horizontal mesh tally of the cyclotron target model.

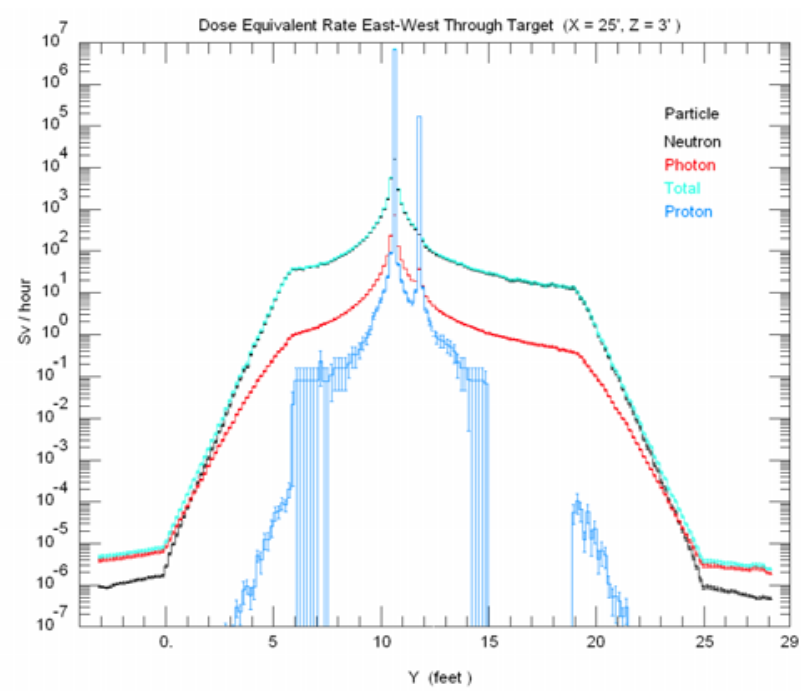

Figure 13. Dose rate profiles from top to bottom through the target in Figures 8-10.

Figure 14 shows the horizontal total dose rate mesh tally of figure 11 in a 3D view where the third dimension is proportional to the tally value. A different color scheme is used than in Figures 8 through 12 in order to better show the decline through the entrance maze.

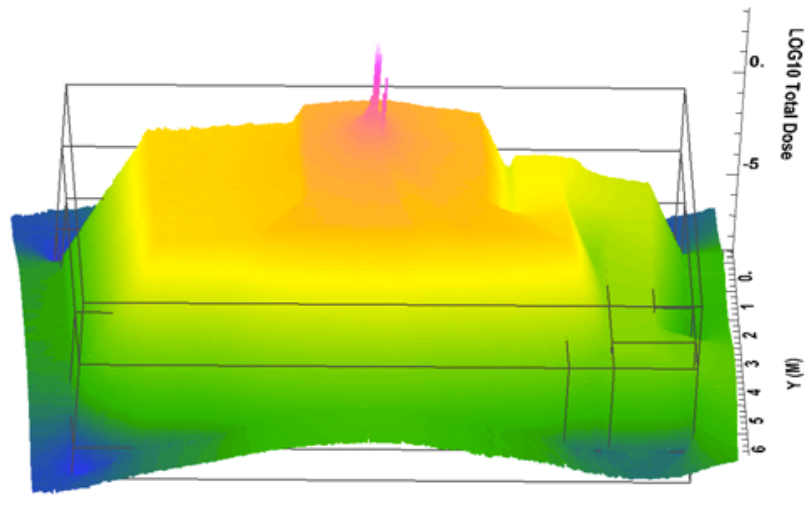

Figure 14. A 3D view of the total dose equivalent rate mesh tally of Figure 11.

\subsection{External target model}

Figures 15 and 16 show the total dose equivalent rate from the external target model. The relative neutron, photon, and proton contributions and the relative errors are similar to those in the cyclotron target model. The peak in the cyclotron room is due to the fraction of protons that strike the collimator.

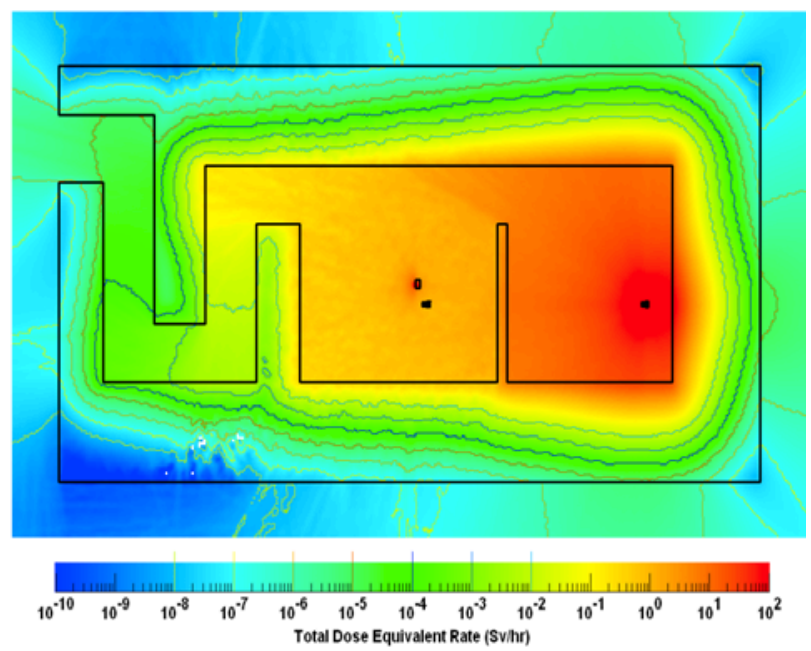

Figure 15. Total dose equivalent rate in the horizontal mesh tally of the external target model.

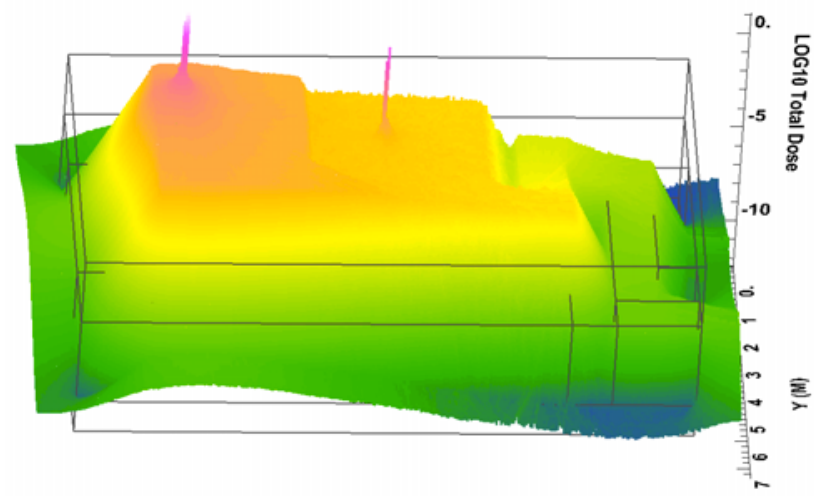

Figure 16. A 3D view of the total dose equivalent rate mesh tally of Figure 15. 


\section{Maze entrance door}

The vault shielding models described above did not include a door at the maze entrance. We made several studies of the attenuation through entrance doors of different thicknesses and compositions. Except for the door and modifications near the door, the geometry, source, and materials are the same as in the cyclotron target model described above. Because the dose rate at the maze entrance is larger using the main cyclotron target, we assumed irradiation of that target for the door calculations.

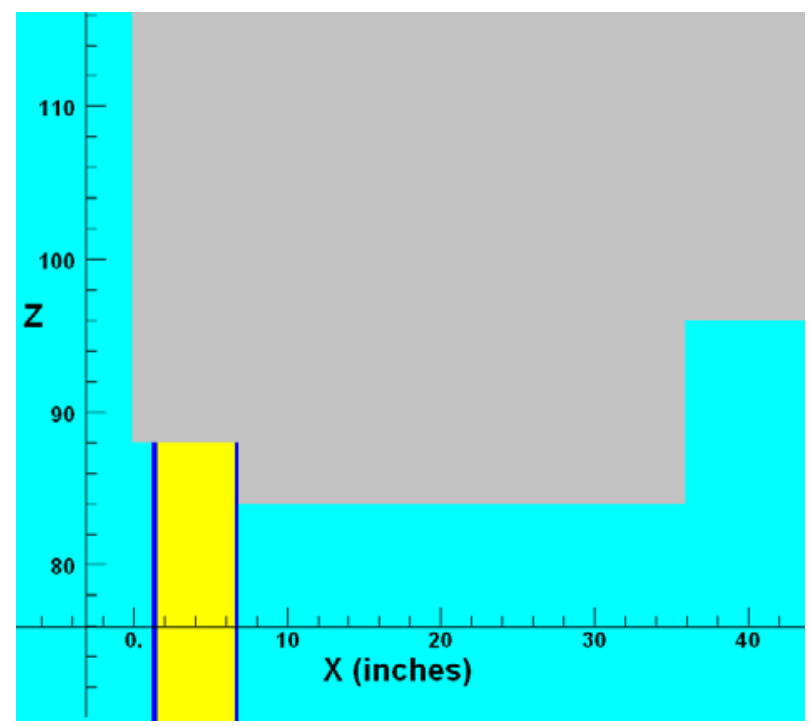

Figure 17. Vertical slice through the top of the maze entrance door. Both scales are in inches.

\subsection{Surface source calculations}

The calculation was made in two stages using the MCNP surface source write (SSW) and surface source read (SSR) features. Without the need to transport particles from the target through the maze, the SSR calculations require much less computation time. The shorter SSR runs permitrd exploration of a number of door designs.

The initial SSW calculation had irradiation of the main target and collimator by the proton beam as the source. All neutrons and protons crossing a surface spanning the air space in the maze coincident with the first inner wall (the green line in Figure 1) were saved to a file. Only outward (upwards in Figure 1) crossings were saved. To reduce calculation time, biasing to enable penetration of the side and rear walls and the ceiling was not included. Biasing through the maze was retained.

The SSW calculation followed 5,761,845 histories, approximately $1 / 10$ of the histories followed in the vault shielding calculations. The small relative errors around the maze entrance found in the previous results justified using the smaller number of histories. The run resulted in a 1.9 GByte surface source file. Comparison of mesh tallies showed that the SSW calculation gave the same results in the cyclotron room and through the maze as the previous shielding calculation.
An SSR run in the same geometry was made to verify that the same results were obtained. Plots of the ratios of the horizontal neutron and photon mesh tallies near the maze entrance showed that the results agree within $20 \%$ in the air volumes downstream of the surface source. A vertical mesh tally outside of the door opening was used to examine any vertical variation of the neutron and photon dose rates. No significant variation was seen.

\subsection{Door model}

The door consists of a polyethylene slab sandwiched between steel plates. It fits into recesses in the outer maze entrance. Figure 17 is a horizontal cut through the model. Figure 18 is a vertical cut through the door. In the figures, concrete is grey, air is light blue, polyethylene is yellow, and steel is dark blue. A horizontal mesh tally extends 25 feet in front of the door.

The side and top recesses are $67 / 8$ inches deep (in the $+\mathrm{X}$ direction in Figures 17 and 18). The east recess (towards the top in Figure 17) extends 9 inches beyond the maze opening. The west recess extends 5 inches beyond the opening. The top overlap is 4 inches high. The door bottom is flush with the concrete floor. The height of the opening is 84 inches, less than the 96 inch height of the maze. Except for the opening through the outer north wall, the height in the maze was kept at 96 inches.

The outer steel layer is $3 / 8$ inch thick; the inner $1 / 4$ inch. A 5 inch thick polyethylene layer was used for the initial calculations. For the 3 inch polyethylene model, the inner thinner steel layer remained as shown and the outer steel layer was moved 2 inches inward $(+\mathrm{X}$ direction).

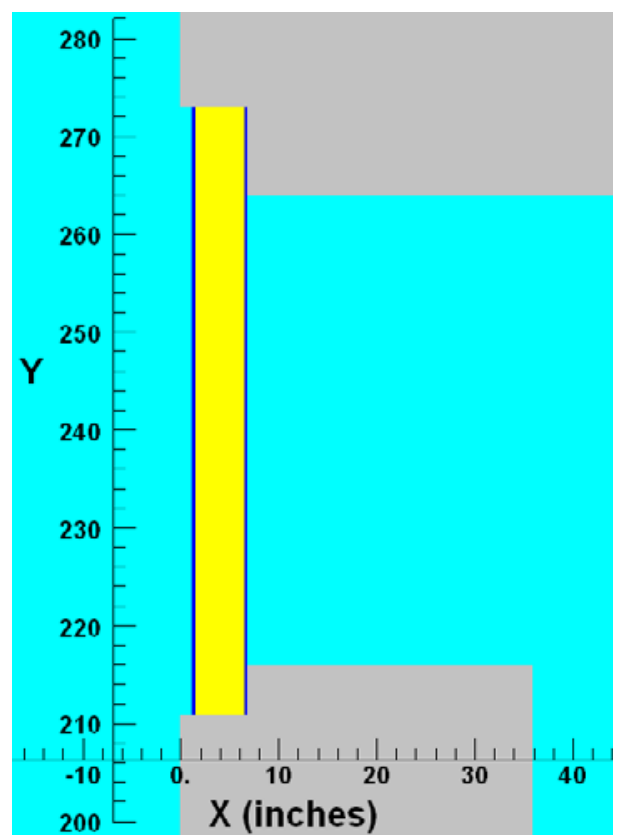

Figure 18. Horizontal slice through the maze entrance and door. Both scales are in inches.

The SSR door calculations followed neutrons and protons from the surface source. These calculations did 
not include penetration of the outer wall other than at the maze opening. The full shielding calculations showed some penetration, especially opposite the second turn in the maze.

\subsection{Polyethylene composition}

A model without a door was run to establish a baseline against which to measure the shielding efficacy of the door options. In the No Door model, the polyethylene and steel layers were replaced by air.

Several compositions were used for polyethylene. The Natural Polyethylene model did not contain any dopants, only $2 / 3 \mathrm{H}$ and $1 / 3 \mathrm{C}$ by number. The Borated Polyethylene model contained $5 \%$ boron by weight added as boric oxide $\left(\mathrm{B}_{2} \mathrm{O}_{3}\right)$. Trace amounts of iron $\left(0.00058 \%\right.$ by weight), sulfate $\left(\mathrm{SO}_{4}, 0.0051 \%\right)$, and water $(1.1 \%)$ found in an analysis of the boric oxide were ignored.

In MCNP, a material constituent can be specified as an element or as the fractions of isotopes making up the element. In the element specification, MCNP chooses the isotopes naturally occurring the element. For boron, we compared runs with natural boron (element specification, Natural B in Figure 11) and with explicit fractions for boron 10 and boron 11 (B10_B11). The results were the same within the statistical uncertainty

\subsection{Maze entrance door results}

The curves in the Figures 19 through 22 show the dose equivalent rates along a north-south line passing through the center of the door (240 inches in Figure 18). The peaks at $91.44 \mathrm{~cm}$ mark the location of the surface source. The curves to the right (larger $\mathrm{X}$ ) of the peak should be ignored. The error bars in Figures 19 and 20 are omitted for clarity.

Figures 19 and 20 show the neutron and photon contributions to the dose rate with no door (19) and with a 5 inch borated polyethylene door. Figure 21 shows that boron doping does not change the neutron contribution. Figure 22 shows that the difference between 5 and 3 inch doors is small.

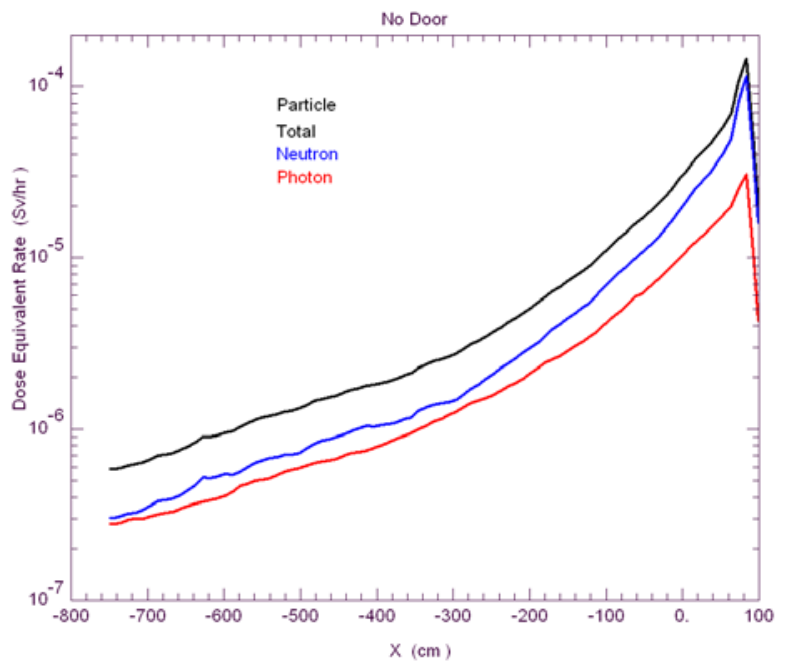

Figure 19. Dose equivalent rate profiles with no door.

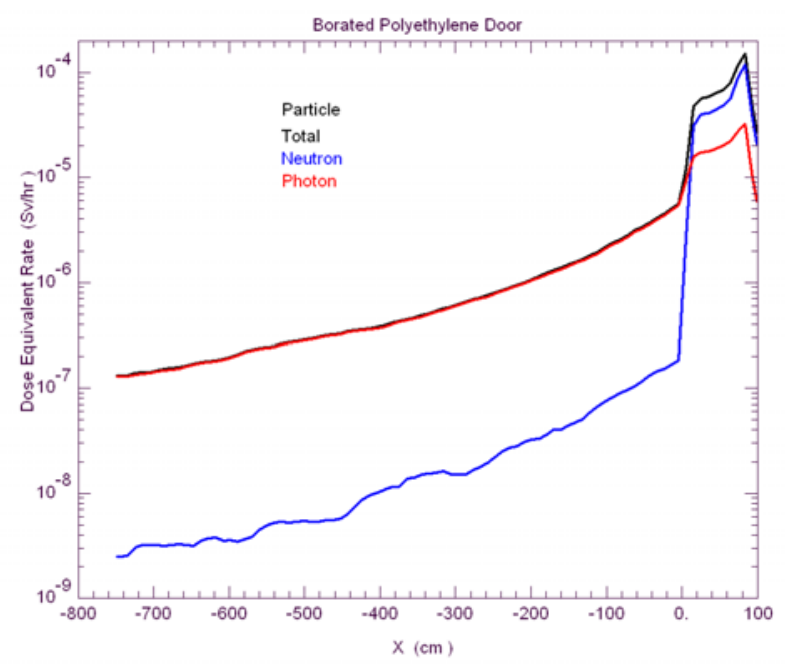

Figure 20. Dose equivalent rate profiles with a borated polyethyline door.

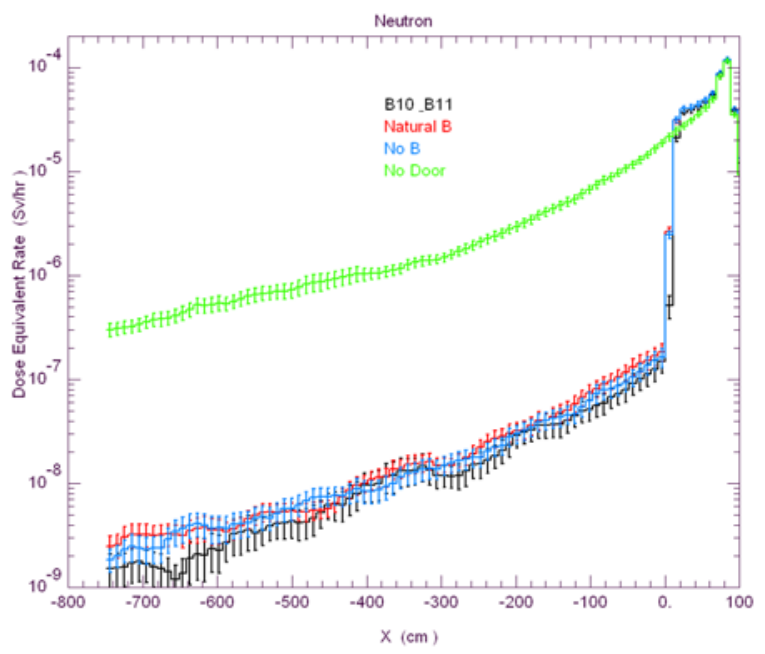

Figure 21. Neutron dose equivalent rate profiles.

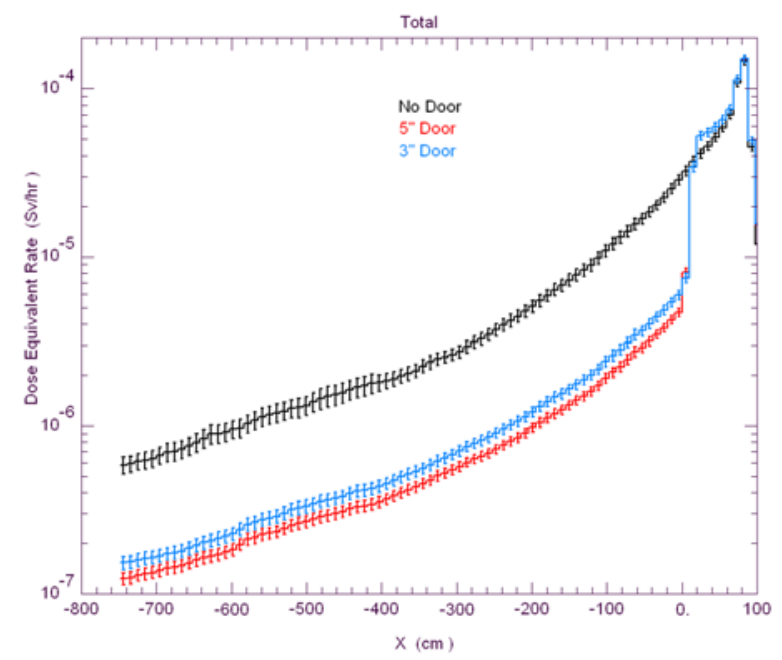

Figure 22. Total dose equivalent rate profiles. 


\section{Steel Rod Penetrations}

A few dozen steel rods were used to hold the concrete forming framework together. These 7/8 inch diameter rods were left in place after the pour. We wanted to ensure that the presence of the rods did not affect the efficacy of the concrete sheilding wall. We prepared a modified model of the cyclotron target room that omitted the entrance maze and the external target room. Eight inches of the wall between the cyclotron room and the external target room and 8 inches of the the opposite wall were retained. The outer walls included 12 rods on each side. Horizontal mesh tallies at the height of the target also covered a row of three rods in each outer wall. Runs with and without the rods showed no difference in the external photon and neutron dose rates.

\section{Conclusion}

We have calculated the dose equivalent rates throughout and just outside of the cyclotron vault. The dose rate external to the vault is less than regulatory limits. In particular, we used the pregnant worker limit of $5 \mathrm{mSv}$ over the course of the pregnancy for occupationally controlled areas and the $1 \mathrm{mSv}$ per year limit for nonoccupational areas. These limits factored in a workload (or use factor) of 0.97 .

Photon measurements were taken above the cyclotron room during irradiation of the main target. The highest dose rates at the roof surface were $0.9 \mu \mathrm{Sv}$ /hour and 0.4 $\mu \mathrm{Sv} /$ hour at 1 meter above the roof. Our calculations predicted 5 and $3 \mu \mathrm{Sv} /$ hour at these locations.

\section{Acknowledgement}

We thank Richard Wentz and Uno Zetterberg of GE Healthcare for their patience in answering numerous questions about the cyclotron and target design and source terms.

\section{References}

1. J. O. Bergstrom, PETTRACE - unshielded machine : Summary of source terms, radiation fields and radwaste production, GE Healthcare Document Ref: RP001181(0.4).doc, (2004)

2. D. Pelowitz (ed.), MCNPX User's Manual, Version 2.7.0, Los Alamos National Laboratory, LA-CP-1100438 (2011). 